\title{
A machine learning predictive model of in-hospital mortality in patients with sepsis complicated by anemia: a retrospective study based on the MIMIC- III database
}

\section{Xiaobin Liu}

Changhai Hospital

\section{Yu Zhao}

Fuzhou University

\section{Yingyi Qin}

Naval Medical University

\section{Dan Wang}

First Affiliated Hospital of Nanchang University

Xi Yin

The Affiliated Zhangjiagang Hospital of Soochow University

\section{Renqi Yao}

Changhai Hospital

\section{Qimin Ma}

Changhai Hospital

\section{Yusong Wang}

Changhai Hospital

Shihui Zhu

Changhai Hospital

\section{Shaolin Ma}

Tongji University Affilliated East Hospital: Tongji University Dongfang Hospital

\section{Zuquan Weng}

Fuzhou University

Feng Zhu ( $\nabla$ alexzhufeng0816@vip.sina.com )

Changhai Hospital

\section{Research}

Keywords: MIMIC-III database, Sepsis, Anemia, Machine Learning and Data Mining 
DOl: https://doi.org/10.21203/rs.3.rs-832364/v1

License: (c) (1) This work is licensed under a Creative Commons Attribution 4.0 International License. Read Full License 


\title{
A machine learning predictive model of in-hospital mortality in patients with sepsis complicated by anemia: a retrospective study based on the MIMIC-III database
}

\author{
Xiaobin Liu',2†, Yu Zhao $2,3 \dagger$, Yingyi Qin ${ }^{4 \dagger}$, Dan Wang ${ }^{5 \dagger}$, Xi Yin ${ }^{6 \dagger}$, Renqi Yao ${ }^{1}$, Qimin Ma1, \\ Yusong Wang ${ }^{1}$, Shihui Zhu ${ }^{1,2}$, Shaolin $\mathrm{Ma}^{7^{*}}$, Zuquan Weng ${ }^{2,3^{*}}$, Feng Zhu ${ }^{7,1,2^{*}}$ \\ *Correspondence: mslin@sohu.com; wengzq@fzu.edu.cn; alexzhufeng0816@vip.sina.com \\ † Xiaobin Liu, Yu Zhao, Yingyi Qin, Dan Wang, Xi Yin contributed equally to this work and are listed as \\ co-first authors. \\ ${ }^{1}$ Burn Institute of PLA, Department of Burns, The First Affiliated Hospital of Naval Medical University, No. \\ 168 Changhai Road, 200433 Yangpu District, Shanghai, China. \\ 2 The Centre for Big Data Research in Burns and Trauma, College of Mathematics and Computer Science, \\ Fuzhou University, No. 2 Xueyuan Road, Minhou County 350108, Fujian, China. \\ Full list of author information is available at the end of the article
}

\section{Abstract}

Backgroud: Patients with sepsis complicated by anemia have a higher risk of mortality. It is clinically important to study the risk factors associated with the prognosis of this disease. The aim of this study was to establish a predictive model of mortality during hospitalization by extracting clinical data from the Medical Information Mart for Intensive Care III (MIMICIII) database.

Methods: The clinical data of patients with sepsis complicated by anemia in the MIMIC-III database were retrospectively analyzed. Indexes were screened by stepwise logistic regression (LR), and machine learning predictive models such as Decision Tree (DT), Random Forests (RF), and eXtreme Gradient Boosting (XGBoost) were developed and compared, identifying advantages and disadvantages of each model.

Results: A total of 13,547 patients with sepsis complicated by anemia were included in the study, among which 1,827 died during hospitalization and 11,720 were still alive at discharge. The preliminary stepwise regression model selected 20 clinical indexes, including Elixhauser comorbidity index, maximum blood urea nitrogen (BUN), and maximum hemoglobin reduction. The predictive models showed good discriminative ability (area under the receiver operating characteristic curve [AUROC]:LR, 0.777; DT, 0.726; RF, 0.788; XGBoost, 0.815) and goodness of fit (area under the precision-recall curve [AUPRC]: LR, 0.350; DT, 0.290; RF, 0.400; XGBoost, 0.428). The Shapley Additive exPlanation (SHAP) values in the XGBoost model showed that Elixhauser comorbidity index, maximum BUN, maximum hemoglobin reduction, ventilator use within 24 hours of admission, and age were significant features for predicting in-hospital mortality in patients with sepsis complicated by anemia.

Conclusions: The XGBoost model had better discrimination ability and goodness of fit when compared with other models. Machine learning algorithms have significant practical value in the development of an early warning system for patients with sepsis complicated by anemia.

Keywords: MIMIC-III database, Sepsis, Anemia, Machine Learning and Data Mining

\section{Background}

Sepsis has been recognized as an important cause of mortality in critically ill patients and is associated with a high incidence of multiple organ dysfunction. It is not rare in clinical practice to find sepsis complicated by anemia, because most patients with severe sepsis for more than 3 days will suffer from anemia [1]. The etiology and pathogenesis of sepsis complicated by anemia are varied. Previous studies have explored the possible causes of anemia, including red blood cell destruction, myelosuppression, decreased erythropoietin, and iatrogenic blood loss [2-6]. However, 
several studies have shown that there may be other causes of anemia during the acute phase of sepsis, including the degradation of the glucose calyx of the vascular endothelial membrane [7,8] leading to the entry of large amounts of tissue fluid into the blood vessels, the dilution of hemoglobin after massive fluid infusion [9], etc.

Moreover, patients with sepsis complicated by anemia have a higher risk of mortality, since severe anemia will aggravate tissue hypoxia, leading to further deterioration of organ function and circulation disorders $[10,11]$. Meanwhile, severe anemia, which often requires blood transfusion, also increases the risk of infection [10]. Hence, it is clinically important to study the risk factors associated with the prognosis of sepsis complicated by anemia. The aim of this study was to establish a predictive model of mortality during hospitalization for patients with sepsis complicated by anemia by extracting clinical data from the Medical Information Mart for Intensive Care III (MIMIC-III) database. Due to the limitations of traditional statistical methods in dealing with covariates and missing values in retrospective studies, we adopted three machine-learning models, namely Decision Tree (DT), Random Forests (RF), and eXtreme Gradient Boosting (XGBoost), and compared their respective advantages and disadvantages. Such tools can be used to explore the risk factors of patients with sepsis complicated by anemia and to predict their mortality risk.

\section{Materials and methods \\ Database}

MIMIC is a large online clinical data set of critically ill patients created by the Massachusetts Institute of Technology (MIT) in 2003 [12,13]. MIMIC-III was released at the end of 2015, and includes 28,000 additional records compared with MIMIC-II; the data were cleansed and proofread to simplify their structure and increase their reliability.

This study was based on the MIMIC-III database, and the project was approved by the Beth Israel

Deaconess Medical Center and the MIT Institutional Review Board. Since all protected private information was identified and removed, individual patient consent was no longer required. As this project is a retrospective and observational study, the clinical data of patients with sepsis were extracted by database management software and language tools; all relevant data were exported, processed, and analyzed by data analysis software. Such analysis does not have any impact on the treatment of patients and has good safety [13]. A Collaborative Institutional Training Initiative (CITI) license was obtained (number 8761695), together with the permission to use the MIMIC-III database in accordance with the relevant regulations.

\section{Inclusion and exclusion criteria}

Inclusion criteria: the diagnosis of sepsis was based on the operational scheme of Sepsis 3.0 [14]. Sepsis was diagnosed in patients suspected to have infection and with sequential organ failure assessment (SOFA) score and quick SOFA (qSOFA) score $\geq 2$. The time when the patient diagnosed with sepsis entered the intensive care unit (ICU) was defined as the admission time. The diagnostic criteria for anemia [15] were hemoglobin $<13.6 \mathrm{~g} / \mathrm{dL}$ for males and $<11.9 \mathrm{~g} / \mathrm{dL}$ for females.

Exclusion criteria: 1 . incomplete hemoglobin data; 2 . not admitted to any ICU; 2 . age $<16$ years; 3. repeated admission to ICU (only the first admission was considered); 4. pregnant or in the perinatal period; 5. total length of ICU stay $<24$ hours.

\section{Variable screening}

Most extracted variables were indexes that might reflect tissue oxygen metabolism and common indexes related to sepsis-associated organ damage. Indexes with missing data rate $\leq 30 \%$ were included in the screening. Clinical and laboratory variables were collected within 24 hours of admission to the ICU. General information collected about the patients included: age; gender; vital signs, such as minimum systolic blood pressure (SBP), minimum diastolic blood pressure (DBP), maximum respiratory rate (RR), and maximum heart rate (HR). The laboratory tests included minimum hemoglobin, maximum hemoglobin reduction, maximum hemoglobin reduction rate, minimum albumin, minimum red blood cell (RBC) count, minimum hematocrit (HCT), minimum mean corpuscular hemoglobin concentration $(\mathrm{MCHC})$, minimum oxygen partial pressure $\left(\mathrm{PO}_{2}\right)$, maximum carbon dioxide partial pressure $\left(\mathrm{PCO}_{2}\right)$, minimum arterial oxygen saturation $\left(\mathrm{SaO}_{2}\right)$, maximum lactate, minimum oxygen saturation with pulse oximetry $\left(\mathrm{SpO}_{2}\right)$, maximum B-type natriuretic peptide (BNP), maximum serum creatinine, maximum blood urea nitrogen(BUN), maximum D-dimer, maximum international normalized ratio (INR), maximum prothrombin time 
(PT), maximum partial thromboplastin time (PTT), minimum platelet count, maximum blood glucose, maximum troponin, and maximum uric acid. The incidence of renal failure, dialysis, ventilator use, sedation, Elixhauser comorbidity index, and vasoactive drugs such as dobutamine, dopamine, epinephrine, norepinephrine, and phenylephrine were also included. In-hospital mortality was observed as the outcome event.

It should be noted that: (1) The relevant indexes within 24 hours include data from 6 hours before admission to the ICU to 24 hours after admission, according to the relevant literature[12,13]; (2) since blood transfusion could have a direct impact on the hemoglobin level, we discarded hemoglobin data taken after transfusion of erythrocytes.

\section{Statistical methods}

Statistical Product and Service Solutions 25 (SPSS 25, International Business Machines Corporation, New York, USA) and R 3.5.2 (R Project for Statistical Computing, Austria, Vienna) were used for data analysis. Data with normal distribution and homogeneity of variance were represented as mean \pm standard deviation $\left(\mathrm{X}^{-} \pm \mathrm{s}\right)$. Data not following the normal distribution were represented as median $(\mathrm{M})$ and quartiles $\left(\mathrm{P}_{25}, \mathrm{P}_{75}\right)$. Count data were presented as numbers (percentages). The Student $t$ test was used to compare normally distributed data. The t' test was adopted when the variance of the data was not uniform. The Mann-Whitney $U$ test was used for data not following the normal distribution. The chi-square test was used to compare the count data between groups. P-values $<0.05$ were considered statistically significant.

Further, we used the Python StatsModels module for feature filtering. In this study, a stepwise logistic regression model with bidirectional method (combination of forward and backward) was used to predict in-hospital mortality and screen variables that might affect outcome events, using the Akaike Information Criterion (AIC). The AIC is a standard method used to evaluate the complexity of a statistical model and to measure how well it fits the data, and it is based on the concept of information entropy. The formula is as follows:

$$
A I C=-2 * \ln (L)+2 * K
$$

where $\mathrm{L}$ is the likelihood value and $\mathrm{K}$ is the number of parameters. The AIC increases with the number of free parameters to improve the optimization of fitting and avoids the occurrence of overfitting as much as possible. Therefore, we selected the minimum AIC information statistics to select the most predictive variables and perform feature screening.

\section{Construction of machine learning models}

The models were built by the Scikit-Learn machine learning library in Python, and the features selected by stepwise logistic regression were used in the machine learning models. Logistic regression (LR) and the DT and RF models were set as the baseline models in this study. Furthermore, the XGBoost model [16] was used to explore the risk factors for patients with sepsis complicated by anemia and to predict the mortality risk.

Given $\mathrm{n}$ samples of patient data and $\mathrm{m}$ features $\boldsymbol{x}_{\boldsymbol{i}}(\boldsymbol{i} \in(0,1,2, \ldots, \boldsymbol{m}))$, the predicted probability $\hat{\boldsymbol{y}}_{\boldsymbol{i}}$ of each patient can be calculated as follows:

$$
\hat{y}_{i}=\sum_{K=1}^{K} \boldsymbol{f}_{k}\left(\boldsymbol{x}_{i}\right), \boldsymbol{f}_{\boldsymbol{k}} \in \mathrm{F}
$$

$\boldsymbol{f}_{\boldsymbol{k}}$ is the prediction fraction of a single decision tree, and $\mathrm{F}$ is the tree space. In order to obtain the optimal solution, the following regularization objectives are optimized:

$$
\begin{gathered}
\mathcal{L}(\phi)=\sum_{i} l\left(\hat{y}_{i}, y_{i}\right)+\sum_{k} \Omega\left(f_{k}\right) \\
\Omega(f)=\gamma T+\frac{1}{2} \lambda\left\|\omega_{i}\right\|^{2}
\end{gathered}
$$

$\boldsymbol{l}$ is the loss function, used to calculate the losses of the predicted value $\boldsymbol{y}_{\boldsymbol{i}}$ and the true value $\boldsymbol{y}_{\boldsymbol{i}}, \boldsymbol{\Omega}$ is the penalty term, and $\boldsymbol{T}$ is the number of leaves. $\boldsymbol{\omega}_{\boldsymbol{i}}$ is the fraction of leaf node $\boldsymbol{i}$, while $\boldsymbol{\gamma}$ and $\boldsymbol{\lambda}$ are the coefficient parameters.

Moreover, in order to make the XGBoost black box model interpretable, the Shapley Additive exPlanation (SHAP) value algorithm [17] was used to interpret the model: the following formula calculates the contribution $\phi_{i}$ of each feature.

$$
\begin{gathered}
\phi_{i}=\sum_{S \subseteq N \backslash\{i\}} \frac{|S| !(M-|S|-1) !}{M !}\left[f_{S \cup\{i\}}\left(x_{S \cup\{i\}}\right)-f_{S}\left(x_{S}\right)\right] \\
f(x)=g\left(z^{\prime}\right)=\phi_{0}+\sum_{i=1}^{M} \phi_{i} \boldsymbol{z}^{\prime}{ }_{i}
\end{gathered}
$$

$\boldsymbol{N}$ is the set of features of the training data set, of dimension $\boldsymbol{M}$, while $\boldsymbol{S}$ is the subset extracted 
from $\boldsymbol{N}$ of dimension $|\boldsymbol{S}| . \boldsymbol{\phi}_{\boldsymbol{i}}$ represents the contribution of feature $\boldsymbol{i}, \boldsymbol{f}(\boldsymbol{x})$ is the prediction value of the decision tree, and $\boldsymbol{z}^{\prime}{ }_{i} \in\{0,1\}^{\boldsymbol{M}}$ is the number of features included in the decision path made by the sample in all of the $\mathrm{M}$ features, with $\boldsymbol{\phi}_{0}$ being constant.

We randomly divided the patient data in a 4:1 ratio, with 4 portions used as the training set and 1 portion as the testing set. The Grid Search Method was used to find the best hyperparameters in the training set, and 5-fold cross validation was used to avoid overfitting. The XGBoost hyperparameter settings are shown in Table S1, while basic information about the training and validation groups is shown in Table S2.

After obtaining the best XGBoost model, the SHAP value algorithm was applied to improve its interpretability, and finally to obtain the ranking by importance of all the characteristics related to the outcome, while distinguishing between protective factors and risk factors. Further, the area under the receiving operating characteristic curve (AUROC) and the area under the precision-recall curve (AUPRC) values were used to evaluate the performance of each model.

\section{Results}

\section{Basic information about the patients}

The patient selection process is represented in Fig. 1

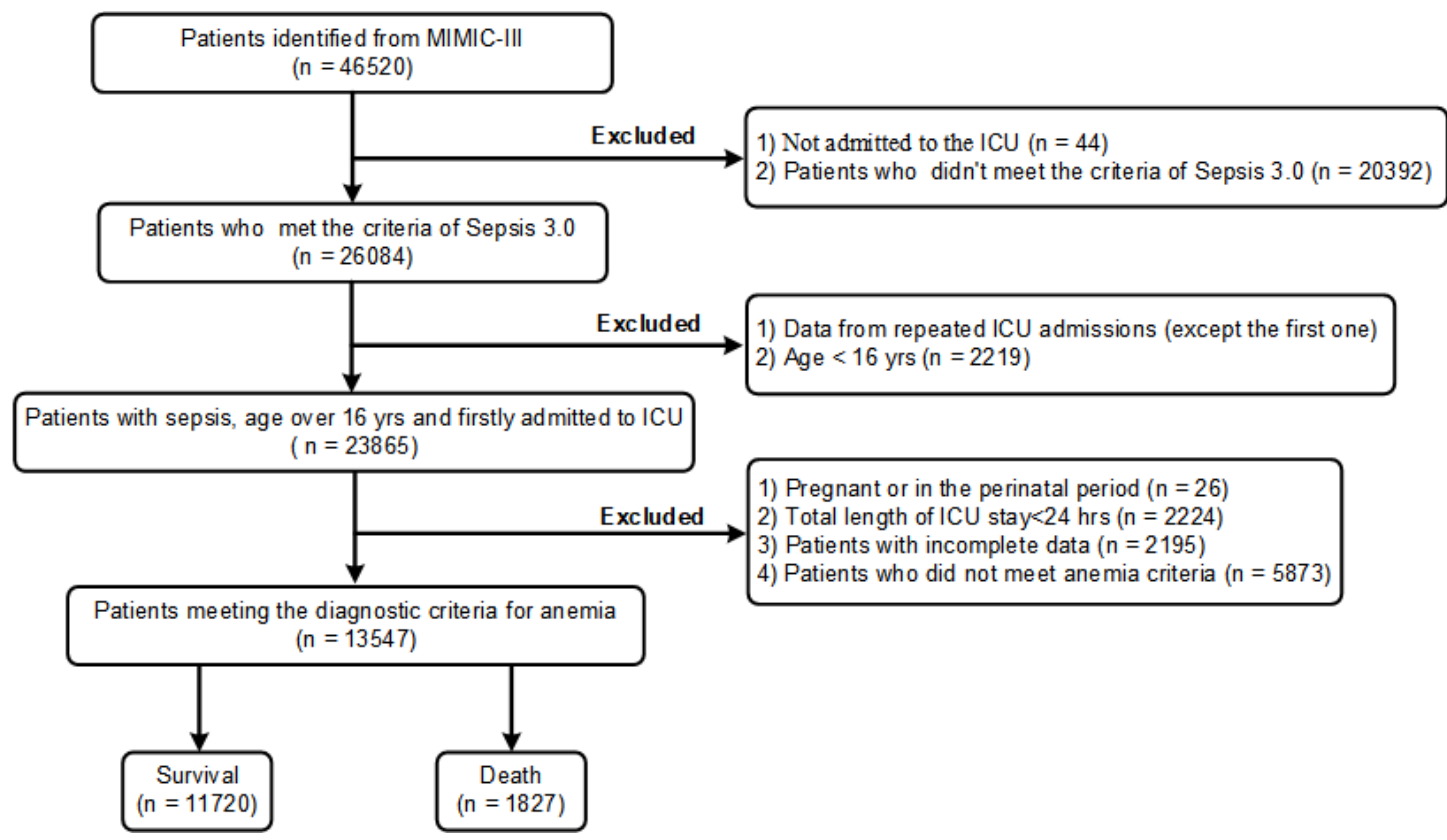

Fig. 1 Screening process of patients with sepsis complicated by anemia

\section{Effect of hemoglobin reduction on in-hospital mortality of patients with sepsis}

LR was used to examine the impact of hemoglobin-related indexes on mortality in patients with sepsis, including hemoglobin at admission (HB_0), maximum hemoglobin reduction within 24 hours (Delta_HB_24h_down_max), and maximum hemoglobin reduction rate within 24 hours (Delta_HB_24h_down_max_R). There were significant differences between the survival and death groups in hemoglobin and maximum hemoglobin reduction $(\mathrm{p}<0.001$, Table 1$)$. The results showed that the decrease of hemoglobin was an independent risk factor for mortality during hospitalization in patients with sepsis.

Table 1 Logistic regression test for hemoglobin related items

\begin{tabular}{lcccc}
\hline Items & OR & $5 \%$ & $95 \%$ & $p$ \\
\hline Hemoglobin at admission & 0.97 & 0.95 & 0.99 & 0.019 \\
Maximum hemoglobin reduction within 24 hours & 0.77 & 0.66 & 0.89 & 0.004
\end{tabular}




\section{Receiver operating characteristic (ROC) curve}

Further analyses were limited to patients with sepsis complicated by anemia. The ROC curves were used to explore the optimal threshold for predicting in-hospital mortality of these patients using hemoglobin-related indexes (Fig. 2). The AUROC of hemoglobin at admission, minimum hemoglobin, maximum hemoglobin reduction, and maximum hemoglobin reduction rate were only $0.580,0.521,0.611$, and 0.606 , respectively. These results imply that the use of hemoglobin-related indexes alone could not accurately predict the in-hospital mortality of patients with sepsis complicated by anemia.

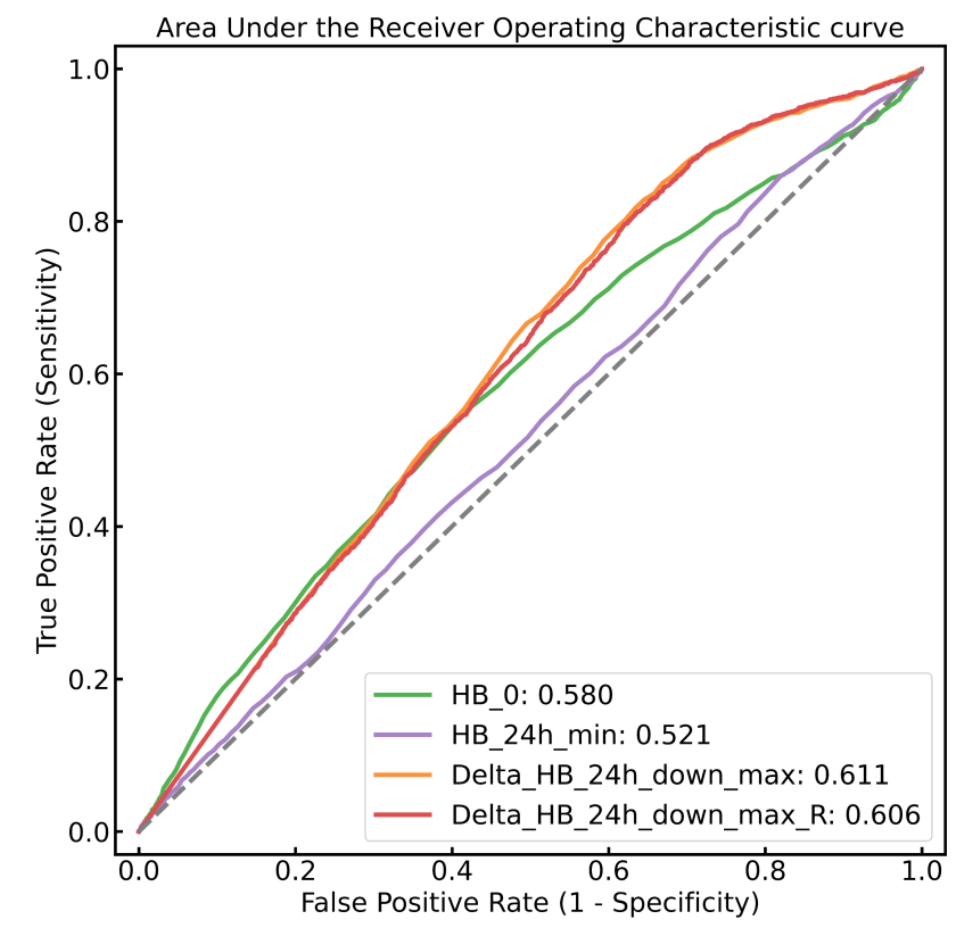

Fig. 2 ROC curves of hemoglobin-related indexes for predicting in-hospital mortality

\section{Baseline data of patients with sepsis complicated by anemia}

A total of 1,827 patients died during hospitalization. The death group was significantly older than the survival group. The proportion of male patients in the death group was significantly lower than that in the survival group. Regarding complications, vasopressors, and special procedures, renal failure, continuous renal replacement therapy (CRRT) use, ventilator use, sedation, dobutamine use, dopamine use, epinephrine use, norepinephrine use, phenylephrine use, and Elixhauser comorbidity index were significantly different between the two groups.

Concerning vital signs, minimum SBP, minimum DBP, maximum HR, maximum RR, and minimum $\mathrm{SpO}_{2}$ were significantly different between the two groups.

In arterial blood gas tests, minimum $\mathrm{PO}_{2}$, maximum $\mathrm{PCO}_{2}$, minimum $\mathrm{SaO}_{2}$, maximum lactic acid were significantly different between the two groups.

Regarding laboratory tests, hemoglobin at admission, minimum hemoglobin, maximum hemoglobin reduction, maximum hemoglobin reduction rate, minimum RBC count, minimum HCT, minimum MCHC, minimum albumin, maximum BNP, maximum creatinine, maximum BUN, maximum blood glucose, maximum troponin, maximum PT, maximum INR, maximum PTT, maximum D-dimer, minimum platelet count were significantly different between the two groups( Table 2). 


\begin{tabular}{|c|c|c|c|}
\hline Items & Survival group & Death group & $p$ \\
\hline \multicolumn{4}{|l|}{ General Information } \\
\hline Age, (years, median $\left.\left[\mathrm{P}_{25}, \mathrm{P}_{75}\right]\right)$ & $69[57,79]$ & $74[61,83]$ & $<0.001$ \\
\hline Gender/Male, $n(\%)$ & $7247(61.83)$ & $1070(58.59)$ & 0.008 \\
\hline \multicolumn{4}{|l|}{ Complications and special procedures } \\
\hline Renal failure, $\mathrm{n}(\%)$ & 2088(17.82) & $413(22.61)$ & $<0.001$ \\
\hline CRRT, n(\%) & $72(0.61)$ & $60(3.28)$ & $<0.001$ \\
\hline Ventilation, $\mathrm{n}(\%)$ & $7117(60.73)$ & $1191(65.19)$ & $<0.001$ \\
\hline Sedation, $\mathrm{n}(\%)$ & $6836(58.33)$ & $1000(54.73)$ & 0.004 \\
\hline $\begin{array}{l}\text { Elixhauser comorbidity index, } \\
\text { (median[}\left[\mathrm{P}_{25}, \mathrm{P}_{75}\right] \text { ) }\end{array}$ & $7[2,13]$ & $13[7,18]$ & $<0.001$ \\
\hline \multicolumn{4}{|l|}{ Vasopressors } \\
\hline Dobutamine, $\mathrm{n}(\%)$ & $173(1.48)$ & $54(2.96)$ & $<0.001$ \\
\hline Dopamine, $\mathrm{n}(\%)$ & $569(4.85)$ & $233(12.75)$ & $<0.001$ \\
\hline Epinephrine, $\mathrm{n}(\%)$ & $779(6.65)$ & $65(3.56)$ & $<0.001$ \\
\hline Norepinephrine, $\mathrm{n}(\%)$ & $1657(14.14)$ & $727(32.95)$ & $<0.001$ \\
\hline Phenylephrine, $\mathrm{n}(\%)$ & $3383(28.87)$ & $430(23.54)$ & $<0.001$ \\
\hline \multicolumn{4}{|l|}{ Vital Signs } \\
\hline Minimum SBP, $\left(\mathrm{mmHg}\right.$, median $\left.\left[\mathrm{P}_{25}, \mathrm{P}_{75}\right]\right)$ & $88[79,97]$ & $82[71,93]$ & $<0.001$ \\
\hline Minimum DBP, $\left(\mathrm{mmHg}, \operatorname{median}\left[\mathrm{P}_{25}, \mathrm{P}_{75}\right]\right)$ & $42[36,48]$ & $39[30,45]$ & $<0.001$ \\
\hline Maximum HR, (bpm, median $\left.\left[\mathrm{P}_{25}, \mathrm{P}_{75}\right]\right)$ & $102[90,116]$ & $111[95,127]$ & $<0.001$ \\
\hline Maximum RR, (bpm, median[ $\left.\left[\mathrm{P}_{25}, \mathrm{P}_{75}\right]\right)$ & $26[23,31]$ & $29[25,34]$ & $<0.001$ \\
\hline Minimum $\mathrm{SpO}_{2},\left(\%\right.$, median $\left.\left[\mathrm{P}_{25}, \mathrm{P}_{75}\right]\right)$ & $91[78,94]$ & $89[74,93]$ & $<0.001$ \\
\hline \multicolumn{4}{|l|}{ Arterial Blood Gas } \\
\hline Minimum $\mathrm{PO}_{2,}\left(\mathrm{mmHg}, \operatorname{median}\left[\mathrm{P}_{25}, \mathrm{P}_{75}\right]\right)$ & $82[60,111]$ & $68[49,95]$ & $<0.001$ \\
\hline $\begin{array}{l}\text { Maximum } \mathrm{PCO}_{2,}(\mathrm{mmHg} \text {, } \\
\left.\text { median }\left[\mathrm{P}_{25}, \mathrm{P}_{75}\right]\right)\end{array}$ & $47[42,53]$ & $45[38,55]$ & $<0.001$ \\
\hline Minimum $\mathrm{SaO}_{2},\left(\%\right.$, median $\left.\left[\mathrm{P}_{25}, \mathrm{P}_{75}\right]\right)$ & $97[95,98]$ & $96[93,98]$ & $<0.001$ \\
\hline $\begin{array}{l}\text { Maximum lactic acid, (mmol/L, } \\
\left.\text { median[ }\left[\mathrm{P}_{25}, \mathrm{P}_{75}\right]\right)\end{array}$ & $2.2[1.5,3.4]$ & $2.8[1.8,5.4]$ & $<0.001$ \\
\hline \multicolumn{4}{|l|}{ Laboratory Tests } \\
\hline $\begin{array}{l}\text { Hemoglobin at admission, }(\mathrm{g} / \mathrm{dL} \text {, } \\
\left.\text { median }\left[\mathrm{P}_{25}, \mathrm{P}_{75}\right]\right)\end{array}$ & $10.9[9.7,11.9]$ & $10.4[9.15,11.4]$ & $<0.001$ \\
\hline $\begin{array}{l}\text { Minimum hemoglobin, (g/dL, } \\
\text { median }\left[\mathrm{P}_{25}, \mathrm{P}_{75}\right] \text { ) }\end{array}$ & $9.1[7.9,10.2]$ & $9.1[8.1,10.3]$ & 0.002 \\
\hline $\begin{array}{l}\text { Maximum hemoglobin reduction, (g/dL, } \\
\left.\text { median[ }\left[\mathrm{P}_{25}, \mathrm{P}_{75}\right]\right)\end{array}$ & $1.2[0.3,2.9]$ & $0.8[0,1.7]$ & $<0.001$ \\
\hline $\begin{array}{l}\text { Maximum hemoglobin reduction rate, }(\% \text {, } \\
\left.\text { median[ }\left[\mathrm{P}_{25}, \mathrm{P}_{75}\right]\right)\end{array}$ & $11.61[3.00,25.93]$ & $7.61[0,16.16]$ & $<0.001$ \\
\hline $\begin{array}{l}\text { Minimum RBC count, }\left(\times 10^{12} / \mathrm{L} \text {, }\right. \\
\left.\text { median }\left[\mathrm{P}_{25}, \mathrm{P}_{75}\right]\right)\end{array}$ & $3.135[2.77,3.50]$ & $3.06[2.69,3.50]$ & $<0.001$ \\
\hline Minimum HCT, $\left(\%\right.$, median $\left.\left[\mathrm{P}_{25}, \mathrm{P}_{75}\right]\right)$ & $26.8[23.1,30.1]$ & $27.4[24.0,30.9]$ & $<0.001$ \\
\hline
\end{tabular}




\begin{tabular}{|c|c|c|c|}
\hline Minimum MCHC, $\left(\mathrm{g} / \mathrm{dL}, \operatorname{median}\left[\mathrm{P}_{25}, \mathrm{P}_{75}\right]\right)$ & $33.4[32.4,34.4]$ & $32.6[31.5,33.7]$ & $<0.001$ \\
\hline Minimum albumin, (g/dL, median $\left.\left[\mathrm{P}_{25}, \mathrm{P}_{75}\right]\right)$ & $3.0[2.5,3.4]$ & $2.7[2.2,3.2]$ & $<0.001$ \\
\hline Maximum BNP, $\left(\mathrm{pg} / \mathrm{mL}\right.$, median $\left.\left[\mathrm{P}_{25}, \mathrm{P}_{75}\right]\right)$ & $4113[1461,10599]$ & $8833[1885,17864]$ & 0.001 \\
\hline $\begin{array}{l}\text { Maximum creatinine, (mg/dL, } \\
\left.\left.\text { median[ } \mathrm{P}_{25}, \mathrm{P}_{75}\right]\right)\end{array}$ & $1.1[0.8,1.7]$ & $1.6[1.0,2.7]$ & $<0.001$ \\
\hline Maximum BUN, (mg/dL, median[ $\left.\left[\mathrm{P}_{25}, \mathrm{P}_{75}\right]\right)$ & $21[15,35]$ & $35[22,56]$ & $<0.001$ \\
\hline $\begin{array}{l}\text { Maximum blood glucose, (mg/dL, } \\
\left.\text { median[ }\left[\mathrm{P}_{25}, \mathrm{P}_{75}\right]\right)\end{array}$ & $168[137,206]$ & $170[132,228]$ & 0.009 \\
\hline $\begin{array}{l}\text { Maximum uric acid, (mg/dL, } \\
\left.\text { median[}\left[\mathrm{P}_{25}, \mathrm{P}_{75}\right]\right)\end{array}$ & $5.8[3.95,8.7]$ & $6.7[4.2,10.5]$ & 0.094 \\
\hline $\begin{array}{l}\text { Maximum troponin, }(\mathrm{ng} / \mathrm{mL} \text {, } \\
\left.\text { median[}\left[\mathrm{P}_{25}, \mathrm{P}_{75}\right]\right)\end{array}$ & $0.10[0.04,0.37]$ & $0.13[0.045,0.445]$ & 0.001 \\
\hline Maximum PT, (s, median $\left.\left[\mathrm{P}_{25}, \mathrm{P}_{75}\right]\right)$ & $15.1[13.8,17.1]$ & $16.3[14.05,21.1]$ & $<0.001$ \\
\hline Maximum INR, (median $\left.\left[\mathrm{P}_{25}, \mathrm{P}_{75}\right]\right)$ & $1.4[1.2,1.7]$ & $1.6[1.3,2.2]$ & $<0.001$ \\
\hline Maximum PTT, (s, median[P $\left.\left.25, \mathrm{P}_{75}\right]\right)$ & $34.4[28.7,44.9]$ & $38.2[29.9,56.95]$ & $<0.001$ \\
\hline $\begin{array}{l}\text { Maximum D-dimer, (ng/mL, } \\
\left.\left.\text { median[P } \mathrm{P}_{25}, \mathrm{P}_{75}\right]\right)\end{array}$ & $2422[1177,5251]$ & $3965[1907,7984.5]$ & $<0.001$ \\
\hline $\begin{array}{l}\text { Minimum platelet count, }\left(\times 10^{9} / \mathrm{L} \text {, }\right. \\
\left.\text { median[ }\left[\mathrm{P}_{25}, \mathrm{P}_{75}\right]\right)\end{array}$ & $162[114,232]$ & $153[83,236]$ & $<0.001$ \\
\hline
\end{tabular}

Variable screening by stepwise logistic regression

Stepwise LR analysis was used to further screen the variables, and 20 variables were included into the final model according to the AIC criterion (Table 3).

Table 3 Stepwise logistic regression model for screening the variables

\begin{tabular}{lllll}
\hline Items & OR & Lower.95 & Upper.95 & $p$ \\
\hline Elixhauser_vanWalRaven & 1.07 & 1.06 & 1.08 & $<0.001$ \\
Urea.nitrogen_24h_max & 1.01 & 1.01 & 1.02 & $<0.001$ \\
Ventilation_24h & 2.39 & 1.96 & 2.90 & $<0.001$ \\
Heart.rate_24h_max & 1.01 & 1.01 & 1.01 & $<0.001$ \\
Respiratory.rate_24h_max & 1.03 & 1.02 & 1.04 & $<0.001$ \\
MCHC_24h_min & 0.86 & 0.82 & 0.90 & $<0.001$ \\
Renal_failure & 0.57 & 0.48 & 0.68 & $<0.001$ \\
Hematocrit_24h_min & 1.08 & 1.05 & 1.11 & $<0.001$ \\
Age & 1.01 & 1.01 & 1.02 & $<0.001$ \\
Red.blood.cells_24h_min & 0.58 & 0.48 & 0.69 & $<0.001$ \\
Platelet.count_24h_min & 1.00 & 1.00 & 1.00 & $<0.001$ \\
PTT_24h_max & 1.01 & 1.00 & 1.01 & $<0.001$ \\
Norepinephrine_24h & 1.40 & 1.20 & 1.64 & $<0.001$ \\
INR_24h_max & 1.07 & 1.04 & 1.11 & $<0.001$ \\
Systolic.blood.pressure_24h_min & 0.99 & 0.99 & 1.00 & $<0.001$ \\
CRRT_24h & 2.19 & 1.38 & 3.49 & $<0.001$ \\
Delta_HB_24h_down_max & 0.58 & 0.42 & 0.81 & 0.001 \\
Sedation_24h & 0.73 & 0.60 & 0.89 & 0.001 \\
Diastolic.blood.pressure_24h_min & 0.99 & 0.98 & 1.00 & 0.011 \\
Epinephrine_24h & 0.71 & 0.50 & 1.00 & 0.047 \\
\hline
\end{tabular}

XGBoost model

First, we adjusted the learning rate (variable learning_rate) and optimum number of iterations 
(sn_estimators) with the Grid Search Method. Then, we established the maximum depth of the decision tree (max_depth), and other parameters. The final hyperparameters are shown in Table S1 in the Supplementary Material.

SHAP is an additive interpretation model. Compared with the traditional feature importance graph of the XGBoost model, its significant advantage is that the influence of each feature can be described by SHAP value with a clear direction, distinguishing positive and negative effects. As shown in Fig. 3, among the variables associated with in-hospital mortality in patients with sepsis complicated by anemia, the top 5 factors were Elixhauser comorbidity index, maximum BUN, maximum hemoglobin reduction, ventilator use, and age. Considering age as an example, red represents older age, while a higher SHAP value indicates a higher risk of mortality, so we concluded that the risk of mortality increases with age.
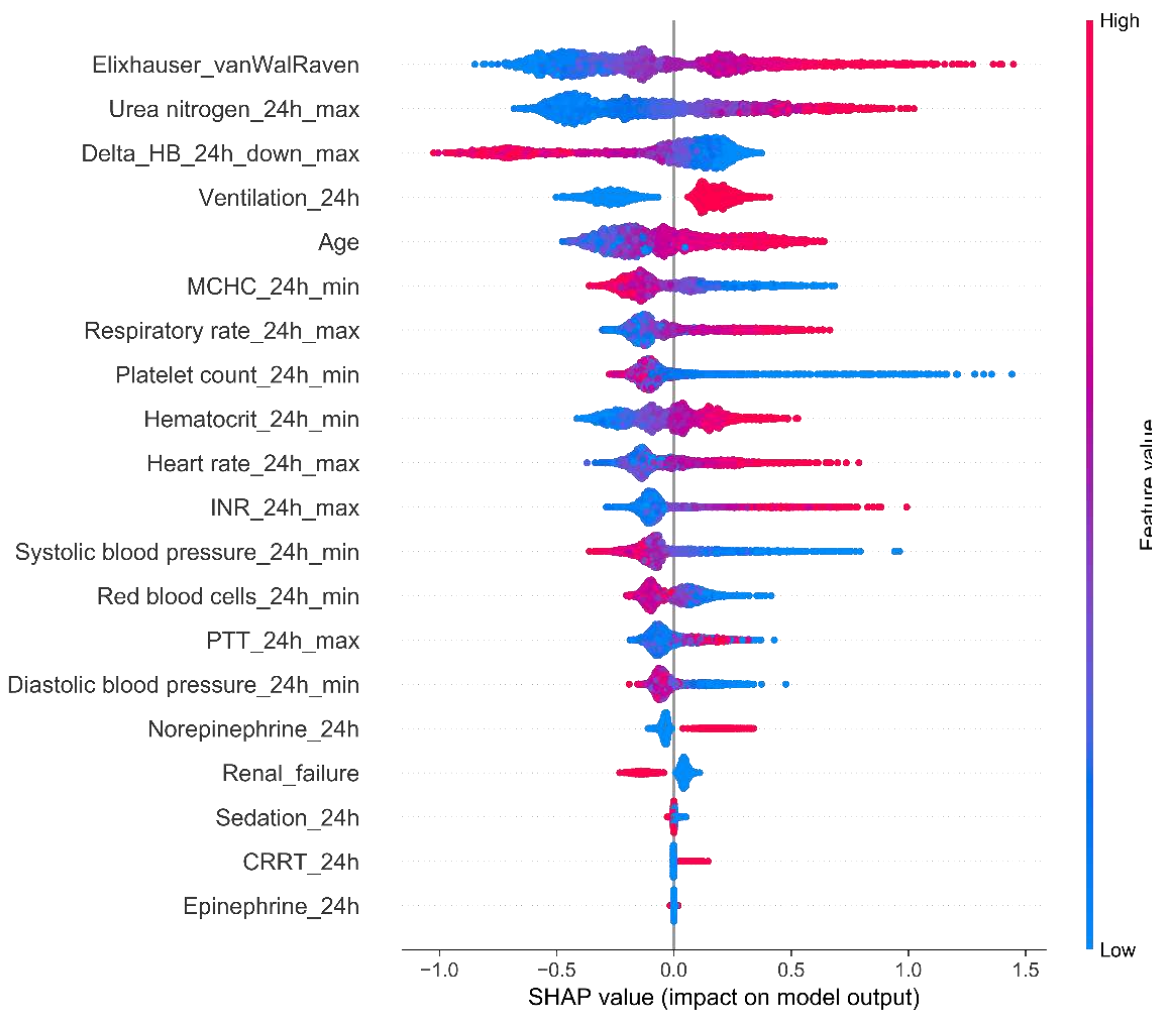

Fig. 3 SHAP values for the XGBoost model. Each point represents a sample. Red (blue) dots represent larger (smaller) values. A larger SHAP value indicates a positive influence on the outcome, and vice versa.

The population was divided into high-risk and low-risk groups according to the median, and the survival curve was plotted. The results showed that the survival probability decreased gradually over time, with significant differences between the two groups ( $p<0.001$, Fig. 4$)$. 

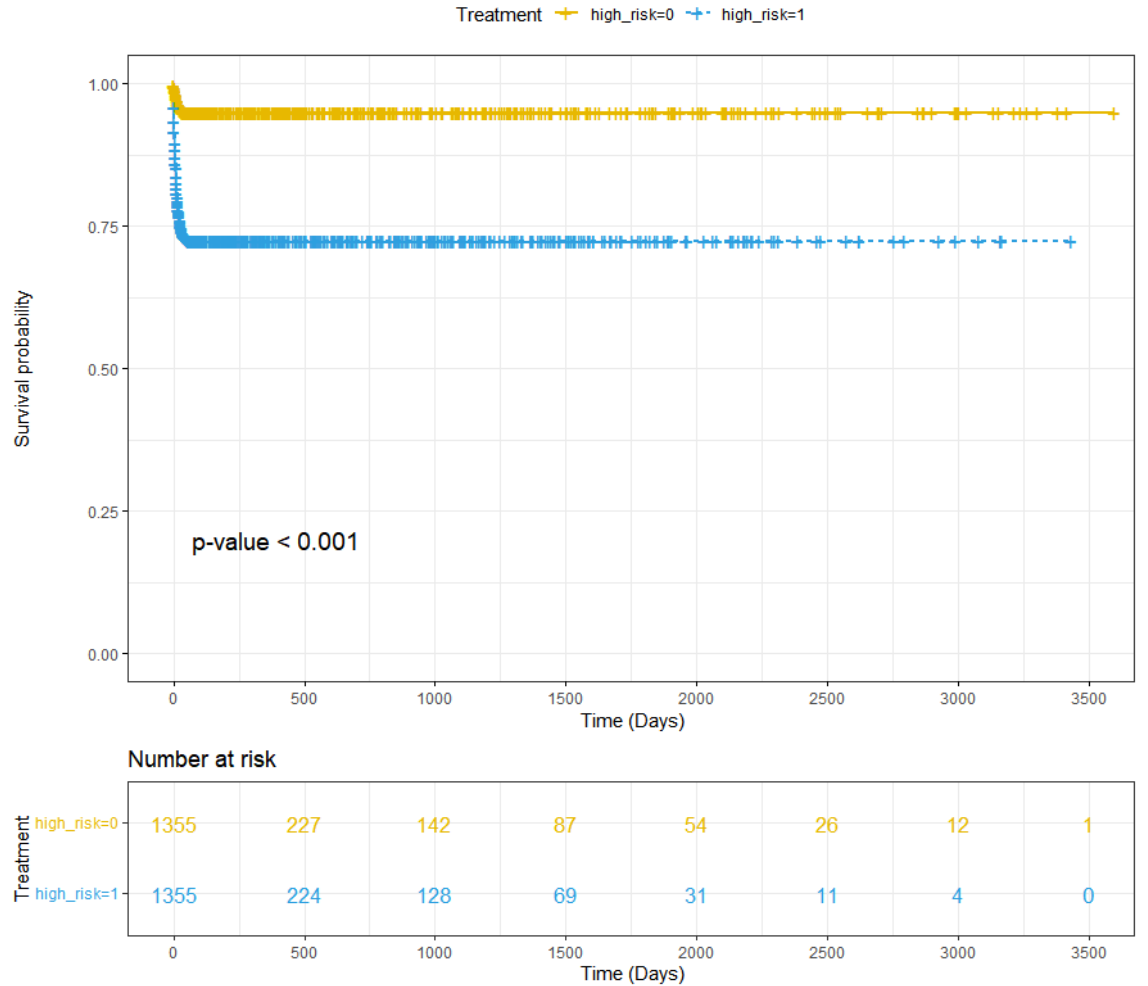

Fig. 4 Kaplan-Meier curves of the high-risk and low-risk groups divided according to the median

\section{Evaluation of the predictive capabilities of different models}

Compared with the stepwise LR, DT, and RF models, the XGBoost model had the highest AUROC value ( 0.815 vs $0.777,0.726$, and 0.788 , respectively), indicating its better discriminating ability (Figure 5A). As shown in Figure 5B, the XGBoost model performed better than stepwise LR, DT, and RF also in terms of AUPRC ( 0.428 vs $0.350,0.290$, and 0.400 , respectively).

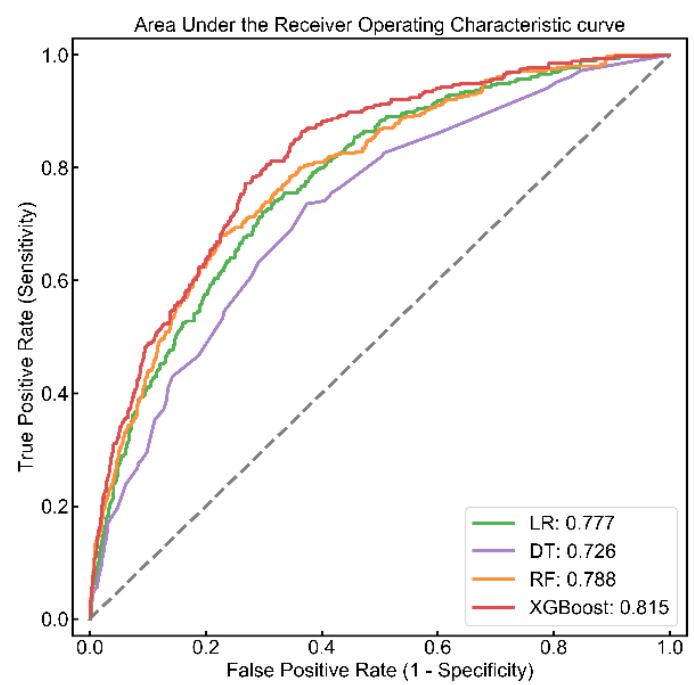

Fig. 5A AUROC of different models

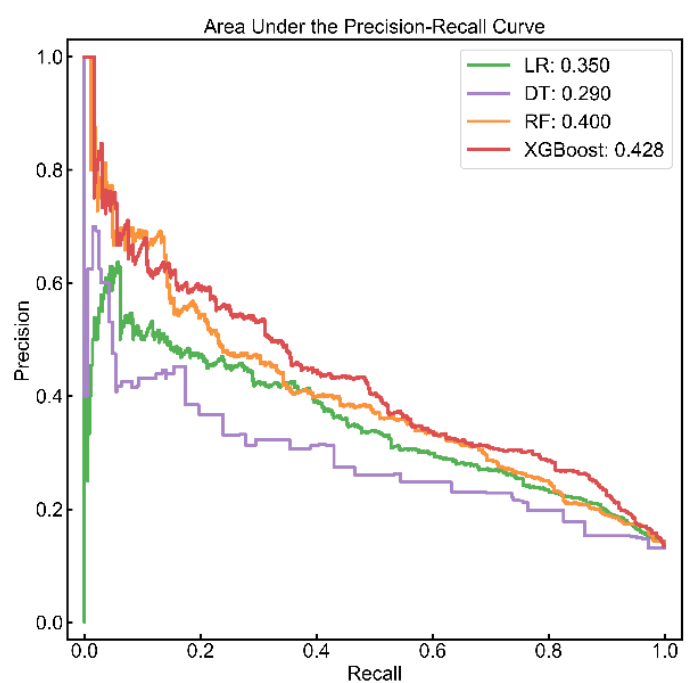

Fig. 5B AUPRC of different models

\section{Discussion}

The etiology and pathogenesis of sepsis complicated by anemia are varied. When infection and immune system dysfunction occur, the erythrocyte cell membrane develops abnormalities due to the damage inflicted by bacteria and immune mechanisms, finally resulting in erythrocyte apoptosis [18]. The cytokines induce activation of mononuclear macrophages and enhanced phagocytosis of 
erythrocytes [19]. However, sepsis is usually accompanied by the formation of a large number of microthrombi. When erythrocytes pass through the thrombi, mechanical damage can also occur and lead to anemia, while patients with sepsis and anemia have more severe tissue hypoxia, are prone to organ function and circulation disorders, and have a higher risk of mortality. Therefore, it is of great clinical significance to study the prognostic risk of sepsis complicated by anemia.

Previous studies have shown that the mortality rate of patients with sepsis is greatly increased when hemoglobin at the time of admission is lower than $8.0 \mathrm{~g} / \mathrm{dL}$ [20]. Our study also confirmed significant differences in the maximum reduction of hemoglobin at the time of admission and the maximum reduction of hemoglobin within 24 hours between patients with sepsis who survived and those who died in the hospital. Thus, we limited the analysis to patients diagnosed with sepsis complicated by anemia and drew ROC curves with hemoglobin-related indexes to build an early warning model of mortality. Regrettably, although a univariable warning model is more convenient and intuitive than those involving multiple variables, it has poor early warning capability. This may be due to the limited ability of a univariable model to fully reveal the overall patient condition.

In addition, while many studies have identified risk factors for mortality in patients with sepsis complicated by anemia during hospitalization, there is still a lack of usable models to accurately predict the clinical outcome of these patients $[15,21,22]$. Machine learning algorithms can help clinical workers to build better prediction models than traditional linear models [23,24]. Consequently, we used a stepwise LR model to screen all clinical data of patients with sepsis complicated by anemia for factors that may have important influence on in-hospital mortality, and constructed DT, RF and XGBoost machine learning predictive models, among which the XGBoost model had the best prediction efficiency. Based on these results, we believe that it is absolutely necessary to use advanced machine learning methods to build predictive models for clinical diseases with complicated pathophysiologic mechanisms and unclear etiopathogenesis.

In our study, we selected 30 covariates related to sepsis with anemia, further screened them by stepwise LR, finally retaining 20 clinical indexes. These indexes were used to construct the DT, RF, and XGBoost models to explore the risk factors and predict the risk of mortality. XGBoost is a kind of gradient lifting tree model, composed of multiple classification and regression trees (CART) and is an example of serial generation model. Each CART divides the patients into two branches according to a certain threshold value of each patient characteristic. After multiple grouping, the end of each CART tree (leaf node) contains patients with the same risk of mortality. The output result of XGBoost is calculated according to the result of the leaf node of each CART. Compared with other traditional machine learning models (LR, DT, or RF), XGBoost is an integrated algorithm based on a tree model, which not only can deal with the problem of data sparsity, but also can learn the nonlinear relationships between features, so as to improve its generalization ability and robustness $[25,26]$. Compared with other models, the XGBoost model has better identification ability and better goodness-of-fit.

In order to make the black box model interpretable, the SHAP algorithm was adopted in this study to interpret it. The SHAP value can be used to interpret not only each patient individually, but also the outcome of all the patients as a whole. By calculating the marginal contribution of each feature of each sample, the feature interpretation of each sample can be deduced from the SHAP value, so as to achieve the effect of local interpretation. Using SHAP values in the XGBoost model, we analyzed the influence of the characteristic values of each clinical index. Through statistics and modeling, we found that Elixhauser comorbidity index, maximum BUN, maximum hemoglobin reduction, ventilator use, and age were the primary predictors of in-hospital mortality within the first 24 hours of ICU admission in patients with sepsis complicated by anemia.

The Elixhauser comorbidity index is a commonly used comprehensive scoring system for evaluating the prognosis of inpatients with underlying diseases [27], often used in studies to reflect disease severity, and is an important confounding factor that needs to be adjusted [28,29]. Indeed, both acute organ damage caused by infection in patients with sepsis and basic diseases such as diabetes, tumors, and renal failure are closely related to the mortality of sepsis [30,31]. The mortality of patients with sepsis is also positively correlated with age [32]. Therefore, it is clinically feasible to use the comorbidity index to determine the risk of mortality of patients with sepsis, consistent with other studies [33-35].

High BUN is a risk factor for mortality in patients with sepsis [36,37]. Indeed, acute kidney injury is not rare in patients with sepsis or septic shock. BUN is an important index of renal function [38], which can reflect the nutritional intake of critically ill patients over a period of time [39]. However, 
while BUN could be easily affected by diet, renal blood flow, high catabolic metabolism, intake of protein or amino acids, as well as intestinal bleeding, hyperthyroidism, and other factors, it is traditionally believed that BUN cannot reflect kidney function better than creatinine [40]. However, a recent study [41] has shown that elevated BUN, rather than serum creatinine, was closely related with increased mortality in critically ill patients whose creatinine was between 0.8 and $1.3 \mathrm{mg} / \mathrm{dL}$. Therefore, whether urea nitrogen is more sensitive to kidney injury than creatinine in a certain group of critically ill patients remains to be elucidated.

Our model, based on large databases, showed that the maximum reduction of hemoglobin within 24 hours in patients with sepsis complicated by anemia was inversely proportional to the risk of mortality, that is, the greater the reduction of hemoglobin, the lower the risk of mortality, which was inconsistent with our previous understanding. Since we collected hemoglobin values before transfusion, the patients did not achieve hemoglobin improvement through transfusion of erythrocytes. In our opinion, the large decrease in hemoglobin may be related to iatrogenic hemodilution caused by the large amount of fluid resuscitation in patients with sepsis at an early stage $[42,43]$. Due to timely and sufficient resuscitation at an early stage, these patients have better prognosis $[44,45]$. In the SHAP value graph, the absolute value of hemoglobin reduction, rather than its proportion, gave a significant contribution, which confirms our view. It is still controversial whether patients with sepsis complicated by anemia should receive blood transfusion [46-48]. Our results suggest that the maximum hemoglobin reduction within 24 hours was not positively associated with the risk of mortality, and that a certain level of decrease in hemoglobin does not affect patient outcomes.

Finally, in the additional files, we further included the top five and top ten contributing indexes into the XGBoost model, with no significant decrease in AUROC and AUPRC, which also proves that the indexes with high SHAP values had indeed good predictive value (Fig. S1 and Fig. S2). In addition, we have established a web page (https://wengzq-lab.cn/sepsismp/) implementing the machine prediction model for researchers to visit and evaluate.

The present study had several important limitations. The MIMIC database contains a large amount of clinical information, and by mining the database some hidden characteristics of diseases that cannot be found by conventional methods can be revealed, which is useful for prognostic purposes and to evaluate drug use or operation risk. However, its main disadvantage is that the data come from a single center in the US, and the majority of the population is of white or black ethnicity. Due to racial differences, the results may not be applicable to all ethnic groups, and in particular to Asian people, who account for a low percentage of the database subjects. Therefore, further evaluation of our machine learning prediction model needs to be performed in other large databases or, preferably, using prospective cohort studies.

\section{Conclusions}

Our prospective study showed that the XGBoost model had better discrimination ability and goodness of fit when compared with other models to accurately predict the mortality in patients with sepsis complicated by anemia during hospitalization. It is necessary to use advanced machine learning methods to build predictive models for clinical diseases with complicated pathophysiologic mechanisms and unclear etiopathogenesis. However, due to racial differences, our results may not be applicable to all ethnic groups.

\section{Key Messages}

- Patients with sepsis complicated by anemia have a higher risk of mortality. It is clinically important to study the risk factors associated with the prognosis of this disease.

- This study established a predictive model of mortality during hospitalization by extracting clinical data from the MIMIC-III database. The top 5 factors which contribute most were Elixhauser comorbidity index, maximum BUN, maximum hemoglobin reduction, ventilator use, and age.

- The XGBoost model had better discrimination ability and goodness of fit when compared with other models. Machine learning algorithms have significant practical value in the development of an early warning system for patients with sepsis complicated by anemia.

\section{Additional files}

Additional files 1: Table S1 XGBoost hyperparameter settings

Additional files 2: Table S2 Baseline data of the training and validation groups 
Additional files 3: Fig. S1 AUROC of XGBoost models with different numbers of features Additional files 4: Fig. S2 AUPRC of XGBoost models with different numbers of features

\begin{abstract}
Abbreviations
Medical Information Mart for Intensive Care, MIMIC; LR, logistic regression; DT, Decision Tree; RF, Random Forests; XGBoost, eXtreme Gradient Boosting; BUN, blood urea nitrogen; AUROC, area under the receiver operating characteristic curve; AUPRC, area under the precision-recall curve; SHAP, Shapley Additive explanation; MIT, Massachusetts Institute of Technology; CITI, Collaborative Institutional Training Initiative; SOFA, sequential organ failure assessment; qSOFA, quick sequential organ failure assessment; SBP, systolic blood pressure; DBP, diastolic blood pressure; RR, respiratory rate; HR, heart rate; RBC, red blood cell; HCT, hematocrit; MCHC, mean corpuscular hemoglobin concentration; $\mathrm{PO}_{2}$, oxygen partial pressure; $\mathrm{PCO}_{2}$, carbon dioxide partial pressure; $\mathrm{SaO}$, arterial oxygen saturation; $\mathrm{SpO}_{2}$, oxygen saturation with pulse oximetry; $\mathrm{BNP}$, B-type natriuretic peptide; INR, international normalized ratio; PT, prothrombin time; PTT, partial thromboplastin time; AIC, Akaike Information Criterion; CRRT, continuous renal replacement therapy; CART, classification and regression trees; IRBs, institutional review boards
\end{abstract}

\title{
Funding
}

This study was funded by Research on in situ skin repair and regeneration based on micro-tissue engineering technology and 3D printing (National Key Research and Development Program of China 2019YFA0110601).

\section{Availability of data and materials}

The datasets generated and/or analyzed during the current study are not publicly available online but could be available from the corresponding author on reasonable request and under prior approval by such committee.

\section{Ethics approval and consent to participate}

The MIMIC-III database has received ethical approval from the institutional review boards (IRBs) at Beth Israel Deaconess Medical Center and Massachusetts Institute of Technology. Because the database does not contain protected health information, a waiver of the requirement for informed consent was included in the IRB approval.

\section{Consent for publication}

Not applicable.

\section{Competing interests}

The authors declare that they have no competing interests.

\section{Authors' contributions}

$\mathrm{XBL}, \mathrm{YZ}$ and $\mathrm{FZ}$ designed the study, drafted the manuscript and take overall responsibility for its content. DW, XY, QM and YSW carried out literature search and data acquisition. YZ, YYQ, RQY and ZQW conducted the search and the statistical analysis. SHZ, SLM and FZ assessed the study eligibility and quality and interpreted the data. All authors contributed to the manuscript and approved the final version to be considered for publication.

\section{Author details}

${ }^{1}$ Burn Institute of PLA, Department of Burns, The First Affiliated Hospital of Naval Medical University, No. 168 Changhai Road, 200433 Yangpu District, Shanghai, China.

${ }^{2}$ The Centre for Big Data Research in Burns and Trauma, College of Mathematics and Computer Science, Fuzhou University, No. 2 Xueyuan Road, Minhou County 350108, Fujian, China.

${ }^{3}$ College of Mathematics and Computer Science, Fuzhou University, No. 2 Xueyuan Road, Minhou County 350108, Fujian, China.

${ }^{4}$ Department of Health Statistics, Naval Medical University, No. 800 Xiangyin Road, Yangpu District 200433, Shanghai, China.

${ }^{5}$ Department of Neurology, The First Affiliated Hospital of Nanchang University, No. 17 Yongwaizheng Street, Nanchang 330006, Jiangxi, China.

${ }^{6}$ Department of Burn and Plastic Surgery, The Affiliated Zhangjiagang Hospital of Soochow University, No. 68 West Jiyang Road, Zhangjiagang 215600, Jiangsu, China.

${ }^{7}$ Department of Critical Care Medicine, Shanghai East Hospital, Tongji University School of Medicine, No. 150 Jimo Road, Pudong New Area District 200120, Shanghai, China. 


\section{Additional files}

Table S1 XGBoost hyperparameter settings

\begin{tabular}{ll}
\hline Hyperparameters & Inhosp_flag \\
\hline learning_rate & 0.05 \\
n_estimators & 100 \\
max_depth & 5 \\
min_child_weight & 0.8 \\
subsample & 0.4 \\
colsample_bytree & 1 \\
gamma & 0.1 \\
reg_alpha & 0.6 \\
reg_lambda & 0.9 \\
\hline
\end{tabular}

Table S2 Baseline data of the training and validation groups

\begin{tabular}{|c|c|c|c|}
\hline Items & Training Group & Validation Group & $p$ \\
\hline $\begin{array}{l}\text { Elixhauser_commorbidity index, (median }\left[\mathrm{P}_{25} \text {, }\right. \\
\left.\mathrm{P}_{75}\right] \text { ) }\end{array}$ & $8[3,14]$ & $8[3,14]$ & 0.890 \\
\hline Maximum BUN, (mg/dL, median $\left.\left[\mathrm{P}_{25}, \mathrm{P}_{75}\right]\right)$ & $23[15,38]$ & $23[15,37]$ & 0.428 \\
\hline $\begin{array}{l}\text { Maximum hemoglobin reduction, }(\mathrm{g} / \mathrm{dL} \text {, } \\
\left.\left.\text { median[ } \mathrm{P}_{25}, \mathrm{P}_{75}\right]\right)\end{array}$ & $1.2[0.3,2.7]$ & $1.1[0.3,2.8]$ & 0.807 \\
\hline Ventilation, $\mathrm{n}(\%)$ & $6650(61.36)$ & $1677(61.18)$ & 0.861 \\
\hline Maximum RR, (bpm, median $\left.\left[\mathrm{P}_{25}, \mathrm{P}_{75}\right]\right)$ & $27[23,31]$ & $27[23,32]$ & 0.483 \\
\hline Maximum HR, (bpm, median[ $\left.\left[\mathrm{P}_{25}, \mathrm{P}_{75}\right]\right)$ & $103[91,118]$ & $103[91,117]$ & 0.998 \\
\hline Minimum MCHC, $\left(\mathrm{g} / \mathrm{dL}\right.$, median[ $\left.\left.\mathrm{P}_{25}, \mathrm{P}_{75}\right]\right)$ & $33.3[32.2,34.3]$ & $33.4[32.3,34.3]$ & 0.110 \\
\hline Renal failure, $\mathrm{n}(\%)$ & $2002(18.47)$ & $499(18.41)$ & 0.942 \\
\hline Age, (years, median $\left.\left[\mathrm{P}_{25}, \mathrm{P}_{75}\right]\right)$ & $69[58,79]$ & $70[57,80]$ & 0.124 \\
\hline Minimum HCT, $\left(\%\right.$, median $\left.\left[\mathrm{P}_{25}, \mathrm{P}_{75}\right]\right)$ & $26.9[23.4,30.2]$ & $26.8[23.2,30.1]$ & 0.442 \\
\hline $\begin{array}{l}\text { Minimum RBC count, }\left(\times 10^{12} / \mathrm{L}\right. \\
\left.\text { median }\left[\mathrm{P}_{25}, \mathrm{P}_{75}\right]\right)\end{array}$ & $3.13[2.76,3.51]$ & $3.10[2.74,3.49]$ & 0.265 \\
\hline $\begin{array}{l}\text { Minimum platelet count, }\left(\times 10^{9} / \mathrm{L}\right. \\
\left.\text { median }\left[\mathrm{P}_{25}, \mathrm{P}_{75}\right]\right)\end{array}$ & $162[110,233]$ & $158[111,227]$ & 0.402 \\
\hline Maximum PTT, (s, median $\left.\left[\mathrm{P}_{25}, \mathrm{P}_{75}\right]\right)$ & $34.9[28.8,46.5]$ & $34.55[29.2,46.1]$ & 0.793 \\
\hline Norepinephrine, $\mathrm{n}(\%)$ & $1822(16.81)$ & $437(16.13)$ & 0.391 \\
\hline Maximum INR, (median $\left.\left[\mathrm{P}_{25}, \mathrm{P}_{75}\right]\right)$ & $1.4[1.2,1.7]^{\prime}$ & $1.4[1.2,1.7]$ & 0.788 \\
\hline Minimum SBP, $\left(\mathrm{mmHg}\right.$, median $\left.\left[\mathrm{P}_{25}, \mathrm{P}_{75}\right]\right)$ & $87[78,97]$ & $87[78,97]$ & 0.718 \\
\hline Sedation, $n(\%)$ & $6285(58.00)$ & $1551(57.23)$ & 0.472 \\
\hline CRRT, $\mathrm{n}(\%)$ & $103(0.95)$ & $29(1.07)$ & 0.571 \\
\hline Minimum DBP, $\left(\mathrm{mmHg}\right.$, median $\left.\left[\mathrm{P}_{25}, \mathrm{P}_{75}\right]\right)$ & $42[36,48]$ & $42[36,48]$ & 0.575 \\
\hline Epinephrine, $\mathrm{n}(\%)$ & $677(6.25)$ & $167(6.16)$ & 0.870 \\
\hline In-hospital mortality, (n,\%) & $1471(13.57)$ & $356(13.14)$ & 0.551 \\
\hline
\end{tabular}

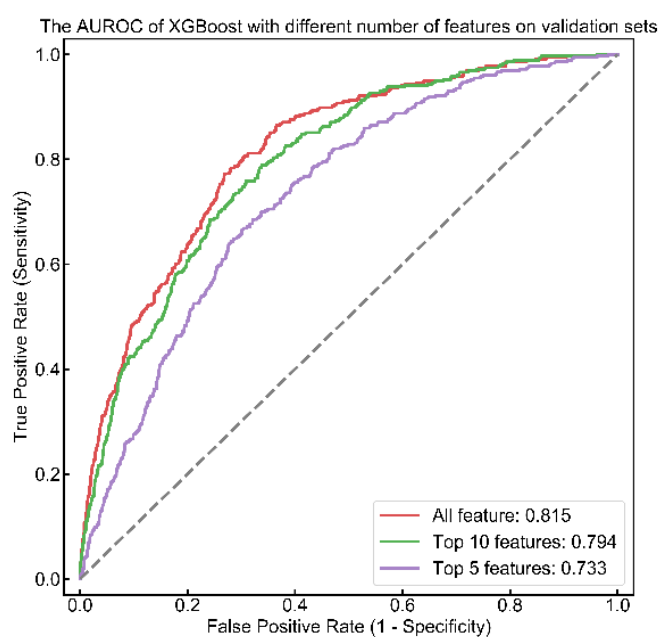

Fig. S1 AUROC of XGBoost models with different numbers of features

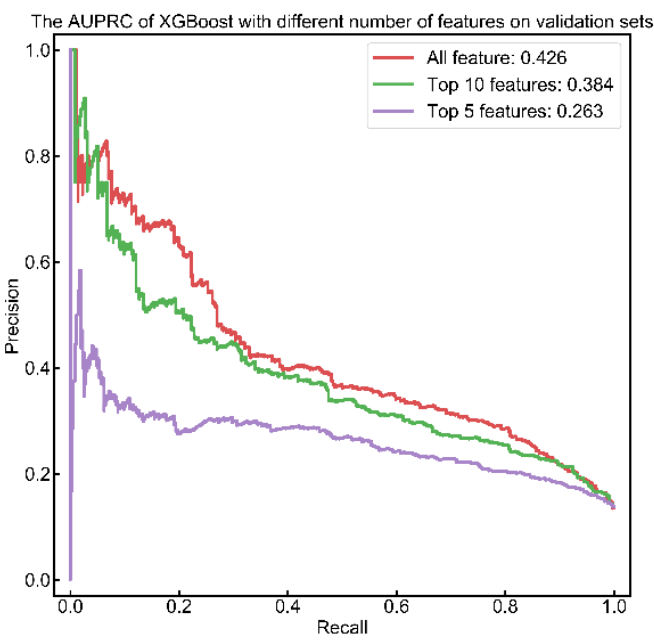

Fig. S2 AUPRC of XGBoost models with different numbers of features 


\section{References:}

1. Corwin HL, Surgenor SD, Gettinger A. Transfusion practice in the critically ill. Crit Care Med. 2003 Dec;31(12 Suppl):S668-71.

2. Bateman RM, Sharpe MD, Singer M, Ellis CG. The Effect of Sepsis on the Erythrocyte. Int J Mol Sci. 2017 Sep 8;18(9):1932. doi: 10.3390/ijms18091932.

3. Zhang H, Rodriguez S, Wang L, Wang S, Serezani H, Kapur R, Cardoso AA, Carlesso N. Sepsis Induces Hematopoietic Stem Cell Exhaustion and Myelosuppression through Distinct Contributions of TRIF and MYD88. Stem Cell Reports. 2016 Jun 14;6(6):940-956.

4. Tamion F, Le Cam-Duchez V, Menard JF, Girault C, Coquerel A, Bonmarchand G. Serum erythropoietin levels in septic shock. Anaesth Intensive Care. 2005 Oct;33(5):578-84.

5. Liu Q, Wu J, Zhang X, Wu X, Zhao Y, Ren J. Iron homeostasis and disorders revisited in the sepsis. Free Radic Biol Med. 2021 Mar;165:1-13.

6. Shander A, Corwin HL. A Narrative Review on Hospital-Acquired Anemia: Keeping Blood where It Belongs. Transfus Med Rev. 2020 Jul;34(3):195-199.

7. Nelson A, Berkestedt I, Schmidtchen A, Ljunggren L, Bodelsson M. Increased levels of glycosaminoglycans during septic shock: relation to mortality and the antibacterial actions of plasma. Shock. 2008 Dec;30(6):623-7.

8. Marechal X, Favory R, Joulin O, Montaigne D, Hassoun S, Decoster B, Zerimech F, Neviere R. Endothelial glycocalyx damage during endotoxemia coincides with microcirculatory dysfunction and vascular oxidative stress. Shock. 2008 May;29(5):572-6.

9. Hale AJ, Ricotta DN, Herzig SJ, William JH, Freed JA. A Quantitative Approach to Dilutional Anemia. J Hematol. 2019 Jun;8(2):86-87.

10. Zimmerman JL. Use of blood products in sepsis: an evidence-based review. Crit Care Med. 2004 Nov;32(11 Suppl):S542-7.

11. Sibbald W.J., Bloos F., Morisaki H. (1996) Sepsis, Anemia, and Tissue O2 Availability. In: Vincent JL. (eds) Yearbook of Intensive Care and Emergency Medicine. Yearbook of Intensive Care and Emergency Medicine, vol 1996. Springer, Berlin, Heidelberg.

12. Johnson, A., Pollard, T., \& Mark, R. (2016). MIMIC-III Clinical Database (version 1.4). PhysioNet.

13. Johnson AE, Pollard TJ, Shen L, Lehman LW, Feng M, Ghassemi M, Moody B, Szolovits P, Celi LA, Mark RG. MIMIC-III, a freely accessible critical care database. Sci Data. 2016 May 24;3:160035.

14. Singer M, Deutschman CS, Seymour CW, Shankar-Hari M, Annane D, Bauer M, Bellomo R, Bernard GR, Chiche JD, Coopersmith CM, Hotchkiss RS, Levy MM, Marshall JC, Martin GS, Opal SM, Rubenfeld GD, van der Poll T, Vincent JL, Angus DC. The Third International Consensus Definitions for Sepsis and Septic Shock (Sepsis-3). JAMA. 2016 Feb 23;315(8):801-10.

15. Adeli K, Raizman JE, Chen Y, Higgins V, Nieuwesteeg M, Abdelhaleem M, Wong SL, Blais D. Complex biological profile of hematologic markers across pediatric, adult, and geriatric ages: establishment of robust pediatric and adult reference intervals on the basis of the Canadian Health Measures Survey. Clin Chem. 2015 Aug;61(8):1075-86.

16. Chen T, Guestrin C. Xgboost: A scalable tree boosting system. Proceedings of the 22nd acm sigkdd international conference on knowledge discovery and data mining. 2016: 785-794.

17. Lundberg S M, Erion G G, Lee S I. Consistent individualized feature attribution for tree ensembles. arXiv preprint arXiv:1802.03888, 2018.

18. Kempe DS, Akel A, Lang PA, Hermle T, Biswas R, Muresanu J, Friedrich B, Dreischer P, Wolz C, Schumacher U, Peschel A, Götz F, Döring G, Wieder T, Gulbins E, Lang F. Suicidal erythrocyte death in sepsis. J Mol Med (Berl). 2007 Mar;85(3):273-81.

19. Iba T, Levy JH. Inflammation and thrombosis: roles of neutrophils, platelets and endothelial cells and their interactions in thrombus formation during sepsis. J Thromb Haemost. 2018 Feb;16(2):231-241.

20. Qi D, Peng M. Early Hemoglobin Status as a Predictor of Long-Term Mortality for Sepsis Patients in Intensive Care Units. Shock. 2021 Feb 1;55(2):215-223.

21. Nguyen BV, Bota DP, Mélot C, Vincent JL. Time course of hemoglobin concentrations in nonbleeding intensive care unit patients. Crit Care Med. 2003 Feb;31(2):406-10.

22. Hayden SJ, Albert TJ, Watkins TR, Swenson ER. Anemia in critical illness: insights into etiology, consequences, and management. Am J Respir Crit Care Med. 2012 May 15;185(10):1049-57.

23. Kang JS, Lee C, Song W, Choo W, Lee S, Lee S, Han Y, Bassi C, Salvia R, Marchegiani G, Wolfgang CL, He J, Blair AB, Kluger MD, Su GH, Kim SC, Song KB, Yamamoto M, Higuchi R, Hatori T, Yang CY, Yamaue H, Hirono S, Satoi S, Fujii T, Hirano S, Lou W, Hashimoto Y, Shimizu Y, Del Chiaro M, Valente R, Lohr M, Choi DW, Choi SH, Heo JS, Motoi F, Matsumoto I, Lee WJ, Kang CM, Shyr YM, Wang SE, Han HS, Yoon YS, Besselink MG, van Huijgevoort NCM, Sho M, Nagano H, Kim SG, Honda G, Yang Y, Yu HC, Do Yang J, Chung JC, Nagakawa Y, Seo HI, Choi YJ, Byun Y, Kim H, Kwon W, Park T, Jang JY. Risk prediction for malignant intraductal papillary mucinous neoplasm of the pancreas: logistic regression versus machine learning. Sci Rep. 2020 Nov 18;10(1):20140.

24. Panesar SS, D'Souza RN, Yeh FC, Fernandez-Miranda JC. Machine Learning Versus Logistic Regression Methods for 2-Year Mortality Prognostication in a Small, Heterogeneous Glioma Database. World Neurosurg X. 2019 Jan 24;2:100012.

25. Kong G, Lin K, Hu Y. Using machine learning methods to predict in-hospital mortality of sepsis patients in the ICU. BMC Med Inform Decis Mak. 2020 Oct 2;20(1):251.

26. Jiang H, Mao H, Lu H, Lin P, Garry W, Lu H, Yang G, Rainer TH, Chen X. Machine learning-based models to support decision-making in emergency department triage for patients with suspected cardiovascular disease. Int J Med Inform. 2021 Jan;145:104326.

27. van Walraven C, Austin PC, Jennings A, Quan H, Forster AJ. A modification of the Elixhauser comorbidity measures into a point system for hospital death using administrative data. Med Care. 2009 Jun;47(6):626-33.

28. Eguia E, Cobb AN, Baker MS, Joyce C, Gilbert E, Gonzalez R, Afshar M, Churpek MM. Risk factors for infection and evaluation of Sepsis-3 in patients with trauma. Am J Surg. 2019 Nov;218(5):851-857.

29. Oh SY, Cho S, Kim GH, Jang EJ, Choi S, Lee H, Ryu HG. Incidence and Outcomes of Sepsis in Korea: A Nationwide Cohort Study From 2007 to 2016. Crit Care Med. 2019 Dec;47(12):e993-e998.

30. Schuetz P, Castro P, Shapiro NI. Diabetes and sepsis: preclinical findings and clinical relevance. Diabetes Care. 2011 Mar;34(3):771-8. 
31. Schrier RW, Wang W. Acute renal failure and sepsis. N Engl J Med. 2004 Jul 8;351(2):159-69.

32. Drechsler S, Weixelbaumer K, Raeven P, Jafarmadar M, Khadem A, van Griensven M, Bahrami S, Osuchowski MF. Relationship between age/gender-induced survival changes and the magnitude of inflammatory activation and organ dysfunction in post-traumatic sepsis. PLoS One. 2012;7(12):e51457.

33. Oh TK, Song IA. Quality of life after sepsis and its association with mortality among sepsis survivors in South Korea: A population level cohort study. J Crit Care. 2021 Aug;64:193-198.

34. Mohr NM, Dick-Perez R, Ahmed A, Harland KK, Shane D, Miller D, Miyake C, Kannedy L, Fuller BM, Torner JC. Emergency department charges may be associated with mortality in patients with severe sepsis and septic shock: a cohort study. BMC Emerg Med. 2018 Dec 29;18(1):62.

35. Kuye I, Anand V, Klompas M, Chan C, Kadri SS, Rhee C. Prevalence and Clinical Characteristics of Patients With Sepsis Discharge Diagnosis Codes and Short Lengths of Stay in U.S. Hospitals. Crit Care Explor. 2021 Mar 16;3(3):e0373.

36. Chaudhary K, Vaid A, Duffy Á, Paranjpe I, Jaladanki S, Paranjpe M, Johnson K, Gokhale A, Pattharanitima P, Chauhan K, O'Hagan R, Van Vleck T, Coca SG, Cooper R, Glicksberg B, Bottinger EP, Chan L, Nadkarni GN. Utilization of Deep Learning for Subphenotype Identification in Sepsis-Associated Acute Kidney Injury. Clin J Am Soc Nephrol. 2020 Nov 6;15(11):1557-1565.

37. Peerapornratana S, Manrique-Caballero CL, Gómez H, Kellum JA. Acute kidney injury from sepsis: current concepts, epidemiology, pathophysiology, prevention and treatment. Kidney Int. 2019 Nov;96(5):1083-1099.

38. Ronco C, Bellomo R, Kellum JA. Acute kidney injury. Lancet. 2019 Nov 23;394(10212):1949-1964.

39. Shavit L, Lifschitz M, Galperin I. Influence of enteric nutrition on blood urea nitrogen (BUN) in very old patients with chronic kidney disease (CKD). Arch Gerontol Geriatr. 2012 Jan-Feb;54(1):228-31.

40. Baum N, Dichoso CC, Carlton CE. Blood urea nitrogen and serum creatinine. Physiology and interpretations. Urology. 1975 May;5(5):583-8.

41. Beier K, Eppanapally S, Bazick HS, Chang D, Mahadevappa K, Gibbons FK, Christopher KB. Elevation of blood urea nitrogen is predictive of long-term mortality in critically ill patients independent of "normal" creatinine. Crit Care Med. 2011 Feb;39(2):305-13.

42. Perel A, Javidroozi M, Shander A. Blood Transfusion in Sepsis and latrogenic Hemodilution. Am J Crit Care. 2018 Nov;27(6):442-443.

43. Perel A. latrogenic hemodilution: a possible cause for avoidable blood transfusions? Crit Care. 2017 Nov 25;21(1):291.

44. Howell MD, Davis AM. Management of Sepsis and Septic Shock. JAMA. 2017 Feb 28;317(8):847-848.

45. Angus DC, van der Poll T. Severe sepsis and septic shock. N Engl J Med. 2013 Aug 29;369(9):840-51.

46. Chan YL, Han ST, Li CH, Wu CC, Chen KF. Transfusion of Red Blood Cells to Patients with Sepsis. Int J Mol Sci. 2017 Sep 11;18(9):1946.

47. Vincent JL, Baron JF, Reinhart K, Gattinoni L, Thijs L, Webb A, Meier-Hellmann A, Nollet G, Peres-Bota D; ABC (Anemia and Blood Transfusion in Critical Care) Investigators. Anemia and blood transfusion in critically ill patients. JAMA. 2002 Sep 25;288(12):1499-507.

48. Leal-Noval SR, Muñoz-Gómez M, Jiménez-Sánchez M, Cayuela A, Leal-Romero M, Puppo-Moreno A, Enamorado $\mathrm{J}$, Arellano-Orden V. Red blood cell transfusion in non-bleeding critically ill patients with moderate anemia: is there a benefit? Intensive Care Med. 2013 Mar;39(3):445-53. 


\section{Supplementary Files}

This is a list of supplementary files associated with this preprint. Click to download.

- Fig.S1AUROCofXGBoostmodels.png

- Fig.S2AUPRCofXGBoostmodels.png

- TableS1XGBoosthyperparametersettings.docx

- TableS2Baselinedataofthetandvgroups.docx 